\title{
USE OF THE MOLECULAR-KINETIC CONCEPTS AT INTRODUCING THE EQUATION OF THE FIRST LAW OF THERMODYNAMICS
}

\author{
Vladimir V. Ryndin \\ Department of Mechanics \\ S. Toraigyrov State University of Pavlodar \\ 140007, 64 Lomov Str., Pavlodar, REPUBLIC OF KAZAKHSTAN
}

\begin{abstract}
The methods of energy transfer and transformation in macroscopic systems are of importance in various areas of science and technology, such as power and heat engineering, chemistry, aerospace technologies, mechanical and biomedical engineering, etc. That is why the present work is topical. Its main target is application of the molecular-kinetic concepts at introducing the equation of the first law of thermodynamics. When presenting this law in textbooks, some troubles appear in differentiating between energy (energy change) and heat or work as well as between heat and work. Therefore the present paper contains a critical analysis of the methods of introducing miscellaneous equations of energy for a closed system and flux, as well as the concepts of heat, work and energy in the scientific literature. By using mathematical manipulations of the Newton's second law written for each single microparticle, a transition is made to the law of energy change (known as the first law of thermodynamics) of all microparticles of a system. Based on the molecular-kinetic concepts, rigorous definitions of such physical quantities as heat and work are formulated.
\end{abstract}

AMS Subject Classification: 74A15, 80A10

Key Words: the first law of thermodynamics, the Newton's second law, energy, heat, momentum, momentum flux

$\begin{array}{lrc}\text { Received: } & 2017-08-04 & \text { (C) } 2017 \text { Academic Publications, Ltd. } \\ \text { Revised: } & 2017-10-30 & \text { url: www.acadpubl.eu }\end{array}$




\section{Introduction}

The thermodynamics is based on some empirical laws irredundant to the fundamental laws of the Newtonian mechanics. One of such laws is the first law of thermodynamics (FLT). Since Clausius has written the book "The Mechanical Theory of Heat", almost all thermodynamics textbooks were written in the same way. At first, the concepts of heat and work are introduced as known from physics. Then the Clausius equation of the first law (principle) of thermodynamics, $d Q=d U+d W$, is written (postulated). From this "the existence" of internal energy (IE) as a derived quantity of heat and work is proved. FLT is formulated as the law of energy conservation and transformation.

At such presentation of FLT in the textbooks, various difficulties appear when differentiating between energy (energy change) and heat or work as well as between heat and work. As a result, energy is defined through the concept of work and IE is defined as the sum (difference) of heat and work, while heat is defined as "a method, form of energy transfer" (i.e., heat is defined not as a physical quantity but as another concept called "form of energy transfer").

Thereby, to obtain the FLT equations and analyze the quantities entering into those equations, a phenomenological method is used in thermodynamics. On the one hand, this method does not concern internal matter structure. On the other hand, it is based on some empirical laws that are not related to the kinetic theory of matter structure. Because of this thermodynamics is considered a phenomenological science (exploring outer sides of phenomena).

However, there are no reasons to draw a sharp boundary between the phenomenological and mechanistic (exploring the interior of phenomena) theories (sciences). No absolutely phenomenological (exploring macro processes) and absolutely mechanistic (exploring micro processes) theories exist. Each theory (science) is mechanistic in relation to some objects and phenomenological in relation to other objects. Every purely empirical science (including thermodynamics) is restricted in its possibilities and its progress without attracting the molecular concepts of matter structure. In this connection many founders of thermodynamics (Clausius, Boltzmann, Planck, etc.) sought to attach a definite molecular sense to such thermodynamic quantities as temperature, heat capacity, entropy, IE, etc. by expressing them in terms of kinetic energy of molecules.

The first works on application of the molecular-kinetic concepts in a specific calculation of some quantities were strongly criticized by reviewers. For example, the known physical formula $p=\frac{2}{3} N_{V} \bar{E}_{k}$ (relating pressure with a number of molecules per unit volume and kinetic energy of translational motion of a 
single molecule) obtained in 1845 by Waterston was rejected by two reviewers (members of the Royal Society of London) and buried in the files of the Society. (In 1891 Lord Rayleigh, while writing his books on The Theory of Sound, searched the literature and came across this paper. After reading it he had it published with a special introduction written by him.) Such was this sad episode.

The prevalent opinion about consistency and completeness of statement of FLT cannot be considered well-grounded. Strict interconnection between heat, work and energy can be established only directly at consideration of FLT rather than before it.

An importance of this work is not only in introducing a method to obtain equation for FLT from application of the Newtonian mechanics to microparticles of matter, but in rigorous substantiation of the proposed concepts of heat, work and IE.

\section{Materials and Methods}

A review of methods of introducing equations of energy and concepts of heat, work and energy in thermodynamics. The FLT equations for "macroscopically stationary body"

$$
\delta Q=\mathrm{d} U+\delta W=\mathrm{d} U+p \mathrm{~d} V, \delta q=\mathrm{d} u+p \mathrm{~d} v
$$

and for flux

$$
\delta Q=\mathrm{d} E+\sum \delta W_{i}=\mathrm{d} E_{\mathrm{k}}+\mathrm{d} E_{\mathrm{p}}+\mathrm{d} U+\sum \delta W_{i} .
$$

are used in thermodynamics. The FLT Eq. (1) obtained by Clausius in 1850 is read as follows: heat $\delta Q$ supplied to a system is used to change IE $(d U)$ and to do work $(\delta W)$. At such an approach a need arises for interpretation of the quantities entering into the energy balance equations: heat, work, energy. Each author makes this independently and uses original literal notation. Following are several examples.

Borgnakke and Sonnag (2009) propose, first of all, the concepts of work $\delta W=P \mathrm{~d} V$ and heat [1]. The symbol $\delta$ is used to denote inexact differentials, as opposed to the symbol d of exact differential. When considering cycles, the "existence" of energy as a difference of heat and work, $\mathrm{d} E=\delta Q-\delta W$, is being proved. FLT for a flux (Eq. (2)) is written as

$$
\mathrm{d} E=\mathrm{d} U+\mathrm{d}(\mathrm{KE})+\mathrm{d}(\mathrm{PE})=\delta Q-\delta W
$$


Callen (1985) proposes the concept of work on volume change $d W_{M}=$ $-P d V[2]$. Heat is considered as difference $d Q=d U-d W_{M}$ of IE change and work change. The infinitesimal quantities $d W_{M}$ and $d Q$ are called imperfect differentials. The FLT Eq. (1) is written as

$$
d Q=d U+P d V .
$$

Hoyst and Poniewierski (2012) propose the concept of work as $d W=-p d V$. The difference $Q=\Delta U-W$ is called heat [3]. Heat is a form of energy transfer. FLT is written as

$$
d U=đ Q+d W+đ Z,
$$

where the symbols $d W, d Q$ and $d Z$ are used to designate infinitesimal quantities of work, heat and chemical work, respectively. Kittel (1973) writes the FLT equation for an open system as [4]:

$$
\mathrm{d} U=D Q+\mu \mathrm{d} N-p \mathrm{~d} V=D Q+D W_{x}+D W .
$$

A change of system IE is a sum of three contributions: $D Q$ (due to energy transfer through thermal contact with a reservoir), $D W_{x}$ (due to passing of particles from a reservoir) and $D W=-p d V$ (due to the work made by a piston over a system).

Kondepudi and Prigogine (2015) write the FLT equation for a closed system as [5]:

$$
\mathrm{d} U=\mathrm{d} Q+\mathrm{d} W=\mathrm{d} Q-p \mathrm{~d} V,
$$

and for an open system as

$$
\mathrm{d} U=\mathrm{d} Q+\mathrm{d} W+\mathrm{d} U_{\text {matter }},
$$

where $d U_{\text {matter }}$ is an addition to IE change through transport of matter. Lavenda (2010) gives the FLT equation in the specific case [6]:

$$
\mathrm{d} Q=m c_{\mathrm{v}} \mathrm{d} T+p \mathrm{~d} V .
$$

Moran et. al. (2014) propose the concept of work as $\delta W=\mathbf{F} \cdot \mathrm{d} \mathbf{s}=p \mathrm{~d} V$ [7]. The differentials of work $(\delta W)$ and heat $(\delta Q)$ are regarded as inexact differentials. The energy balance Eq. (2) is

$$
\Delta E=\Delta \mathrm{KE}+\Delta \mathrm{PE}+\Delta U=Q-W .
$$


Reyf (1972) writes the FLT equation for infinitesimal change of state as [8]:

$$
d \bar{E}=d W+đ Q
$$

where $d \bar{E}$ is infinitesimal change of mean energy; $d W$ and $d Q$ are infinitesimal work and heat, respectively, that are not differences (differentials) of two works and two heats, respectively.

Morales-Rodriguez (2016) immediately proposes the concept of IE $U$ as energy of all molecules of a system, that of work as $d w=-p d V$, and then uses the FLT Eq. (1) as [9]:

$$
\Delta U=q+w
$$

where work $w=-\int_{V_{\mathrm{i}}}^{V_{\mathrm{f}}} p \mathrm{~d} V$. Heat is defined through heat capacity.

Srivastava et. al. (2007) propose at first the concept of system energy as energy of all microparticles of the system. The formulae $d W=p d V$ and $d W=-p d V$ are given for expansion work and compression work, respectively. Therefore, different FLT forms are given for the expansion and compression processes (thus complicating understanding of equations) [10]:

$$
Q-W=\Delta E \text { and } Q+W=\Delta E
$$

Baehr (1977) proposes the concept of work on volume change as $d W^{v}=$ $-p d V$ and gives thermodynamic definition of IE $U$ through the adiabatic work $U_{2}-U_{1}=W_{a d}[11]$. Heat $Q$ is determined from FLT for a rest closed system as energy crossing the system borders as heat, $Q=U_{2}-U_{1}-W_{12}$. The FLT equation for a moving closed system (see Eq. (2)) is written as

$$
Q_{12}+W_{12}=U_{2}-U_{1}+m\left(c_{2}^{2}-c_{1}^{2}\right) / 2+m g\left(z_{2}-z_{1}\right) .
$$

Novikov (1984) proposes the concept of work on volume change $d L=p d V$ and that of heat as $d Q=C_{X} d T$ [12]. The law of energy conservation and transformation is formulated; it is written as $d E=d Q-d L$ for a closed system. It is proved that difference of elementary quantities of heat and work is a total differential of system IE: $d U=d Q-d L$. For a closed system, FLT is written as

$$
\mathrm{d} Q=\mathrm{d} U+\mathrm{d} L=\mathrm{d} U+p \mathrm{~d} V
$$

and for an open system as

$$
\mathrm{d} Q=\mathrm{d} U+\mathrm{d} L-\sum \phi_{i} \mathrm{~d} G
$$


where $\phi_{i}$ is the chemical potential of the $i$-th component.

Kirillin et al. (1983) write FLT in general view [13]:

$$
\mathrm{d} Q=\mathrm{d} U+\mathrm{d} L=\mathrm{d} U+p \mathrm{~d} V
$$

and for a flux

$$
\mathrm{d} q=\mathrm{d} i+w \mathrm{~d} w+g \mathrm{~d} h+\mathrm{d} l_{\mathrm{tw}}+\mathrm{d} l_{\mathrm{frw}},
$$

where $i$ is enthalpy, $w$ is the flux velocity, $h$ is height, $l_{\text {tech }}$ is technical work and $l_{f r}$ is friction work in a flux.

Krutov et al. (1991) write equation of the energy conservation law in the most general view through the different actions $Q_{k}[14]$ :

$$
\Delta U=\sum_{k} Q_{k} .
$$

Heat $Q$ is considered as the quantity of thermal action; the rest actions are called work $L$. Volume work of strain is considered as $d L=p d V$. The FLT Eq. (1) for a thermal deformation system is written as

$$
\mathrm{d} U=\mathrm{d} Q-\mathrm{d} L .
$$

Bazarov (1991) proposes the concepts of work and heat as $\delta W=p d V$ and $\delta Q=T d S$, respectively [15]. In this case it is noted that these differential expressions are not total differentials of some function of the system state parameters. Because of this elementary work and heat are designated $\delta W$ and $\delta Q$, respectively, rather than $d W$ and $d Q$. The FLT Eq. (1) is written as

$$
\delta Q=\mathrm{d} U+\delta W .
$$

Mazur (2003) proposes the concept of work on volume change as $\delta l^{v}=p d v$ and the FLT Eq. (1) as [16]:

$$
\delta q=\mathrm{d} u+\delta l^{v}=\mathrm{d} u+p d v .
$$

Bazhin et al. (2004) note that work of the system may be related to mechanical expansion work $P d V$ as well to other its types (e.g., work opposite to electric field). Therefore, in the general case $\delta W=P d V+\delta W^{\prime}$. Here $\delta W^{\prime}$ is work in excess to the expansion work that is usually called effective work. The first principle of thermodynamics in the absence of effective work is written as $[17]$ 


$$
\mathrm{d} U=\delta Q-\delta W=\delta Q-P \mathrm{~d} V
$$

Lukanin et al. (2000) define specific work and specific heat as $d l=p d v$ and $d q=T d s$, respectively [18]. Concerning a closed thermodynamic system, FLT is written as

$$
\mathrm{d} U=\mathrm{d} Q-\mathrm{d} L=\mathrm{d} Q-p \mathrm{~d} V .
$$

Vukalovich and Novikov (1968) present an expression for work on volume change as $d L=p d V$. The difference of elementary quantities of heat and work produced by a system $(d Q-d L)$ is a total differential of some function of system state; it is called IE and is designated as $U$ [19]:

$$
\mathrm{d} Q=\mathrm{d} U+\mathrm{d} L=\mathrm{d} U+p \mathrm{~d} V .
$$

This equation is analytic expression of the first principle (law) of thermodynamics.

Krutov et al. (1986) propose the concept of elementary work on volume change as $d L=p d V$; an infinitesimal heat quantity is designated as $d Q$. The FLT equation for an isolated system is [20]

$$
\mathrm{d} Q=\mathrm{d} U+\mathrm{d} L .
$$

As a working mass moves through moving canals between the turbine blades, the external work is expended for the work $d L_{s c h}=d(p V)$ of working mass displacement through the canal (state change) and for the technical work $d L_{\text {tech }}$. As a result, the FLT Eq. (26) for open systems is written as

$$
\mathrm{d} Q=\mathrm{d} U+\mathrm{d} E_{\mathrm{k}}+\mathrm{d} E_{\mathrm{p}}+\mathrm{d} L_{\mathrm{SC}}+\mathrm{d} L_{\mathrm{tw}} .
$$

For a detailed analysis of methods of obtaining the FLT equations for closed and open systems as well as for flux see the monographs by Ryndin (2015) and (2007) [21] and [22].

\section{Results and Discussion}

Different authors use various symbols to designate elementary quantities of heat and work: $d Q$ and $d W$ - in the monographs by Kondepudi and Prigogine (2015), Lavenda (2010), Morales-Rodriguez (2016), Srivastava et. al. (2007), Baehr (1977), Novikov (1984), Kirillin et al. (1983), rutov et al. (1991), Lukanin et 
al. (2000), Vukalovich and Novikov (1968), Krutov et al. (1986); $d Q$ and $d W$ - in the monographs by Callen (1985), Hoyst and Poniewierski (2012), Reyf (1972); $\delta Q$ and $\delta W$ - in the monographs by Borgnakke and Sonnag (2009), Moran et. al. (2014), Bazarov (1991), Mazur (2003), Bazhin et al. (2004), Ryndin (2015), Ryndin (2007); $D Q$ and $D W$ - in the monograph by Kittel (1973).

The special symbols $₫, D$ and $\delta$ are considered as symbols of inexact, imprecise differentials of heat and work to distinguish them from the symbol $d$ (total differential of the state functions). It should be noted that any differential (total, inexact, partial) initially designates a difference, increment ( $d$ is the first letter of the word differentia -difference). The concept of differential was proposed by Leibniz in 1684 as an infinitesimal difference of two adjacent values of a quantity. The elementary quantities of heat and work do not characterize motion (energy) storage in a given state. So there is no reason to talk about their difference, or increment as well as to call them differentials (even "inexact" or "partial") and designate them by differential symbols $d, D$ (to avoid readers disorientation). It seems that one might use the terms "incomplete" ("partial") system energy increments in processes of heat exchange $\succsim E_{\text {heatex }}$ and fulfillment of work $d E_{\text {fulwork }}$ that are equal to the elementary quantities of heat $\delta Q$ and work $\delta W$, respectively. For more details see the monographs by Ryndin $(2015,2007)$ [21] and [22].

Loitsianskii (2003) has noted that symbol $\delta$ should be used to designate small (elementary) quantities, e.g., edge length $\delta x$ of a parallelepiped representing a liquid particle at the moment, contrary to the travel $d x=c_{x} d t$ of a liquid particle in a time $d t$. Therefore the symbol $\delta$ should be used as a symbol of elementary character of heat and work (rather than an "inexact differential"): $\delta Q$ is elementary heat and $\delta W$ is elementary work [23].

All authors are united in choosing the sign of heat Q: if energy (motion) is supplied in the process of heat exchange, then $Q>0$; if it is removed, then $Q<0$. As to the sign of work $W$, there is no common opinion concerning choosing sign. For example, Borgnakke and Sonnag (2009), Moran et. al. (2014), Lavenda (2010), Novikov (1984), Kirillin et al. (1983), Krutov et al. (1986 and 1991), Bazarov (1991), Mazur (2003), Bazhin et al. (2004), Lukanin et al. (2000), Vukalovich and Novikov (1968) consider the work taken from a system (expansion work) as positive: $\delta W=\delta W_{\text {exp.w }}=p \mathrm{~d} V>0$. But Callen (1985), Hoyst and Poniewierski (2012), Kittel (1973), Kondepudi and Prigogine (2015), Reyf (1972), Morales-Rodriguez (2016) and Baehr (1977) consider the work supplied to a system (compression work) as positive: $\delta W=\delta W_{\text {com.w }}=$ $-p \mathrm{~d} V>0$. In accordance with the general theory of writing balance equations 
(the supplied fluxes are considered as positive, while the removed fluxes are considered as negative), it is recommended to consider the supplied work (as well as heat supplied to a system) as positive, so the FLT equation should be written as $\mathrm{d} U=\delta Q+\delta W$.

In spite of the fact that thermodynamics is a phenomenological science, many authors use the molecular-kinetic theory of matter structure (in one way or another) to explain the concepts of energy, heat and work. For example Vukalovich and Novikov (1968) note: "From the viewpoint of the kinetic theory of matter heat is related to motion of particles (of which all bodies are made up) and represents a microphysical form of energy transfer from one body to another through either direct molecular interaction (i.e., energy exchange between randomly moving particles of both bodies) or energy emission by one body and absorption of emitted energy by another one. Contrary to heat, work is a macrophysically ordered form of energy transfer through mutual mechanical action of bodies. Therein lies the qualitative difference between work and heat" [19].

Krutov et al. (1986) note: "Thermal motion is a special form of motion of matter (that differs qualitatively from mechanical motion at which all parts of a body are moving in order) and heat is a form of thermal motion. A totality of energy of thermal motion of all the microparticles of a system and their interaction energy is system's internal energy.

Energy transfer (forms of energy change) is made in two ways: by work $\mathbf{L}$ and heat $Q$. Energy transfer resulting from a macroscopic ordered motion of microparticles is called work; that resulting from exchange of chaotic (undirected) motion of microparticles is called heat exchange, and the quantity of energy transmitted in this case is called quantity of heat, process heat or heat" [20].

When applying the kinetic concepts, the authors often use the same denomination for different categories: motion as attribute of matter, motion forms, processes, ways of transfer of motion (energy) - the real objects of the world and physical quantities - items from the world of ideas (named numbers). For example, heat (the physical quantity $Q$ ) may be considered in the other context as the form of thermal motion or energy exchange. The physical quantities $L$ and $Q$ (items from the world of ideas) are identified with the real objects - the processes (ways) of transfer of motion. It should be noted that such confusion of categories may be found in all works on thermodynamics and physics. For example, Bazhin et al. (2004) note that "the main forms of energy transfer are work $W$ and heat $Q$ " [17]. For criticism of confusion between different philosophical categories when explaining the physical quantities see the monograph 
by Ryndin (2015) [21]. It also follows from the above-mentioned that thermodynamics has no method to obtain the Clausius' energy equations by applying the Newtonian mechanics to quasiparticles of matter. Following is presented a possibility of getting in principle the FLT equation for a gas flux from the molecular-kinetic theory of matter structure and thus making possible to give a strict substantiation of the concepts of heat, work and IE.

Derivation of the FLT equation for a flux on the basis of molecularkinetic concepts of matter structure. Since the concept of force is the basis for the concepts of energy and work, an idea arises as to whether it is possible to pass on to the law of conservation of energy of all microparticles composing a system by mathematical transformations of the Newton's second law written for each separate microparticle. Orir in his book "Fundamental Physics" (1969) [24] pointed to a possibility that the Newton's laws could be derived from the laws of conservation of energy and momentum (and vice versa). The number of really fundamental laws of nature is extremely small, so it is a matter of taste what can be considered the main law.

Guided by the above statement, let us derive the energy balance Eq. (2) for a mobile element of a flux by applying the laws of the classical Newtonian mechanics to the motion of particles of the considered element: microparticles (atoms, electrons, molecules, etc.) and submicroparticles (even smaller microparticles - gravitons, photons, phonons, inertons, etc.) that provide interaction between microparticles at a distance; this interaction is characterized by potential energy. To this end, let us separate a small element of a mobile medium (see Fig. 1) composed of $N$ particles.

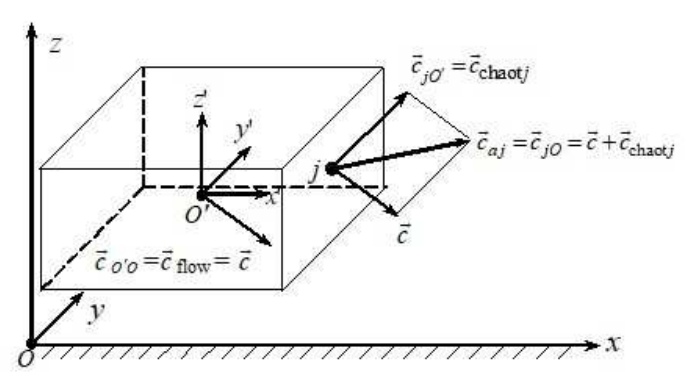

Figure 1: The decomposition of the absolute velocity of particle into components

The absolute (full) velocity $\vec{c}_{a j}=\vec{c}_{j O}$ of the separate $j$-th particle relative to a fixed reference frame $O$ connected with canal walls is a sum of the transport velocity $\vec{c}_{\mathrm{trv}}=\vec{c}_{O^{\prime} O}=\vec{c}_{\text {ordm }}=\vec{c}_{\text {flux }}=\vec{c}$ of the center of inertia $O^{\prime}$ 
of a mobile element of medium relative to the center of the stationary reference frame $O$ (velocity of ordered, directed motion of particles, or flux velocity) and relative velocity $\left(\vec{c}_{j O^{\prime}}=\vec{c}_{\text {chaot } j}\right)$ relative to the center $O^{\prime}$ of the mobile coordinate system that is related to the center of inertia of an element of medium (velocity of chaotic motion of particles):

$$
\vec{c}_{a j}=\vec{c}_{j O}=\vec{c}_{O^{\prime} O}+\vec{c}_{j O}=\vec{c}+\vec{c}_{\text {chaot } j}
$$

Let us pass on to the momentums by multiplying the velocities in Eq. (28) into the mass $m_{j}$ of the $j$-th particle:

$$
\vec{K}_{a j}=m_{j} \vec{c}_{a j}=m_{j} \vec{c}+m_{j \operatorname{chaot} j} .
$$

The momentum of the $j$-th particle changes due to its interaction with other particles. In this case the total momentum increase of the $j$-th particle in a time dt will be equal to decrease of momentums of the particles that have interacted with the $j$-th particle (among those $n$ particles are the system intrinsic particles as well as the environmental particles $\left.\left(n=n_{\text {intr }}+n_{\text {envp }}\right)\right)$ or, putting it otherwise, it will be equal to the sum of elementary momentums transferred due to those interactions:

$$
\mathrm{d} \vec{K}_{a j}=-\sum_{i=1}^{n} \mathrm{~d} \vec{K}_{i j}=\sum_{i=1}^{n} \delta \vec{K}_{i j} .
$$

By dividing the left and right parts of Eq. (30) with time $d t$, we obtain

$$
\mathrm{d} \vec{K}_{a j} / \mathrm{d} t=\sum_{i=1}^{n} \delta \vec{K}_{\mathrm{ij}} / \mathrm{d} t
$$

The ratio between the physical quantity $B$ (that characterizes transfer of matter and motion through the system boundary) and time interval $\delta t=\mathrm{d} t$ (in which that transfer has happened) is usually called a flux $\dot{B}=\delta B / \delta t=\delta B / \mathrm{d} t$ of the above physical quantity (mass flux, energy flux, heat flux, charge flux - electric current strength, etc.). Therefore, the ratio between the elementary momentum $\delta \vec{K}$ of ordered motion (transferred through the system boundary in time interval $d t$ ) and this time interval is nothing more than momentum flux (Landau and Lifshitz, 1988), or a force of the $i$-th particle action on the $j$-th one: $\vec{F}_{i j}=\delta \vec{K}_{i j} / \mathrm{d} t[25]$. Then Eq. (31) becomes the equation of the Newton's second law

$$
\mathrm{d} \vec{K}_{a j} / \mathrm{d} t=\sum_{i=1}^{n} \vec{F}_{i j}
$$


according to which the particle rate of change of momentum is equal to the sum of forces (momentum fluxes) of $\mathrm{n}$ other particles acting on the $j$-th one.

If both parts of Eq. (32) are scalar multiplied by the displacement vector $\mathrm{d} \vec{s}_{j}$ of the $j$-th particle, then we pass on from the momentum flux balance or force balance (if the rate of momentum change is considered as own momentum flux or intrinsic force) to the energy balance (if heat and work are considered as "transition energies"):

$$
\mathrm{d} \vec{K}_{a j}\left(\mathrm{~d} \vec{s}_{a j} / \mathrm{d} t\right)=\sum_{i=1}^{n} \vec{F}_{i j} \mathrm{~d} \vec{s}_{j}
$$

Taking into account (i) that time derivatives of displacements are velocities of absolute, ordered and chaotic motions:

$$
\vec{c}_{a j}=\mathrm{d} \vec{s}_{a j} / \mathrm{d} t=\mathrm{d} \vec{s}_{\text {ord } j} / \mathrm{d} t+\mathrm{d} \vec{s}_{\text {chaot } j} / \mathrm{d} t=\vec{c}+\vec{c}_{\text {chaot } j} .
$$

as well as (ii) Eq. (29) for momentum, we obtain the energy balance Eq. (33) for a separate particle as

$$
\begin{aligned}
\mathrm{d}\left(m_{j} \vec{c}_{a j}\right) \vec{c}_{a j}=\mathrm{d}\left(m_{j} \vec{c}\right) \vec{c}+\mathrm{d}\left(m_{j} \vec{c}\right) \vec{c}_{\text {chaot } j}+\mathrm{d}\left(m_{j} \vec{c}_{\text {chaot } j}\right) \vec{c}+ & \\
& +\mathrm{d}\left(m_{j \text { chaot } j}\right) \vec{c}_{\text {chaot } j}=\sum_{i=1}^{n} \vec{F}_{i j} \mathrm{~d} \vec{s}_{j} .
\end{aligned}
$$

After summing Eq. (35) for all $(N)$ system particles and taking into account that the total momentum of chaotic particle motion is zero (the particle mass is considered constant):

$$
\sum_{j=1}^{N} \vec{c}_{\text {chaot } j} \mathrm{~d}\left(m_{j} \vec{c}\right)=\mathrm{d} \vec{c} \sum_{j=1}^{N} \vec{c}_{\text {chaot } j} m_{j}=0 ; \vec{c} \mathrm{~d} \sum_{j=1}^{N} \vec{c}_{\text {chaot } j} m_{j}=0,
$$

we obtain energy equation for a mobile element of medium (total mass $m=\sum_{j=1}^{N} m_{j}$ :

$$
\sum_{j=1}^{N} \vec{c}_{a j} \mathrm{~d}\left(m_{j} \vec{c}_{a j}\right)=\vec{c} \sum_{j=1}^{N} \mathrm{~d}\left(m_{j} \vec{c}\right)+\sum_{j=1}^{N} \vec{c}_{\text {chaot } j} \mathrm{~d}\left(m_{j} \vec{c}_{\text {chaot } j}\right)=\sum_{j=1}^{N} \sum_{i=1}^{n} \vec{F}_{i j} \mathrm{~d} \vec{s}_{i} .
$$

Let us use the following designations: 


$$
\mathrm{d} E_{\mathrm{k}}=\vec{c} \sum_{j=1}^{N} \mathrm{~d}\left(m_{j} \vec{c}\right)=m \mathrm{~d}\left(c^{2} / 2\right)
$$

a change of kinetic energy of the ordered motion of system particles (flux element of mass $m$ );

$$
\begin{array}{r}
\mathrm{d} U \equiv \mathrm{d} E_{\text {chaot }}=\sum_{j=1}^{N} \vec{c}_{\text {chaot } j} \mathrm{~d}\left(m_{j} \vec{c}_{\text {chaot } j}\right)=\sum_{j=1}^{N} \mathrm{~d}\left(m_{j} c^{2}{ }_{\text {chaot } j}\right) / 2= \\
=\sum_{j=1}^{N} \mathrm{~d} E_{\text {k chaot } j}
\end{array}
$$

a change of flux element internal energy - an energy of chaotic motion of all system own particles (micro- and submicroparticles) relative to their center of inertia (mobile or stationary). For ideal gas the energy of motion of submicroparticles (particles-connectors) that provide interactions between microparticles at a distance is not taken into account, and an internal energy change is equal to a change of kinetic energy of chaotic motion of system atoms and molecules;

$$
\mathrm{d} E_{\text {intr }}=\sum_{j=1}^{N} \vec{c}_{a j} \mathrm{~d}\left(m_{j} \vec{c}_{a j}\right)=\mathrm{d} E_{\mathrm{k}}+\mathrm{d} U
$$

is the change of system own energy - an energy of all microparticles of an assigned element of medium in their absolute motion relative to the canal walls (without a motion energy of particles of the external fields, e.g., gravitational field of the Earth).

At interaction of two system intrinsic particles, the forces of their interaction are equal modulo but opposite in sign. Therefore, the sum of products of these forces by displacements will be zero for all $n_{\text {intr }}$ system intrinsic particles interacting with each other: $\sum_{j=1}^{N} \sum_{i=1}^{n_{\text {intr }}} \vec{F}_{i j} \mathrm{~d} \vec{s}_{i}=0$. Taking into account the designations used and remaining external interactions with $n_{E N V}$ environmental particles, Eq. (40) is

$$
\mathrm{d} E_{\text {intr }}=\mathrm{d} E_{\mathrm{k}}+\mathrm{d} U=\sum_{j=1}^{N} \sum_{i=1}^{n_{\mathrm{ENV}}} \vec{F}_{i j} \mathrm{~d} \vec{s}_{i}
$$


The summed unidirectional actions of separate microscopic forces (momentum fluxes) cause appearance of macroscopic forces (pressure, friction, etc.) acting on an environmental element. Separate microscopic movements may be unidirectional (thus leading to macroscopic displacements), while other forces and movements remain microscopic. Therefore the right part of Eq. (41) can be divided into two summands by using the method of calculation of forces and displacements; each of them characterizes motion energy $E$ (the term "energy" has categorial multiple meaning: it means motion itself as well as the physical quantity $E-a$ quantitative characteristic of motion) transferred by macroscopic $\left(\delta E_{\text {macro }}\right)$ and microscopic $\left(\delta E_{\text {micro }}\right)$ ways, or partial system intrinsic energy gains in these processes:

$$
\begin{aligned}
\sum_{j=1}^{N} \sum_{i=1}^{n_{E N V}} \vec{F}_{i j} \mathrm{~d} \vec{s}_{i} & =\left(\sum_{j=1}^{N} \sum_{i=1}^{n_{E N V}} \vec{F}_{i j} \mathrm{~d} \vec{s}_{\mathrm{i}}\right)_{\text {macro }}+\left(\sum_{j=1}^{N} \sum_{i=1}^{n_{E N V}} \vec{F}_{i j} \mathrm{~d} \vec{s}_{\mathrm{i}}\right)_{\text {micro }}= \\
& =\delta E_{\text {macro }}+\delta E_{\text {micro }}=d\left(E_{\text {intr }}\right)_{\text {macro }}+d\left(E_{\text {intr }}\right)_{\text {micro }} .
\end{aligned}
$$

The energy of transferred motion can be calculated as a product of macroscopic forces (macroscopic momentum fluxes) and displacements. It is commonly called as macroscopic work or simply work (the products of microscopic forces and displacements of separate particles are called microworks):

$$
\begin{aligned}
& \sum_{i=1}^{n_{\mathrm{ENV}}} \delta W^{\prime}{ }_{i}=\left(\sum_{j=1}^{N} \sum_{i=1}^{n_{E N V}} \vec{F}_{i j} \mathrm{~d} \vec{s}_{i}\right)_{\text {macro }}= \\
&=\sum_{i=1}^{n_{E N V}} \vec{F}_{i} \mathrm{~d} \vec{s}_{i}=\delta E_{\text {macro }}=d\left(E_{\text {intr }}\right)_{\text {macro }} .
\end{aligned}
$$

Here the upper stroke means that the work is external (with the plus sign) because it is made by external forces on the system and is equal to loss of the energy of external bodies (gain of the system energy).

If action of microscopic forces does not lead to appearance of macroscopic displacements, then the changes of both the system energy and transferred energy are calculated by other methods (without considering forces and displacements). The part of system energy change (transferred energy) that cannot be calculated using forces and displacements is usually called heat (heat quantity):

$$
\delta Q^{\mathrm{e}}=\left(\sum_{j=1}^{N} \sum_{i=1}^{n_{E N V}} \vec{F}_{i j} \mathrm{~d} \vec{s}_{i}\right)_{\text {micro }}=\delta E_{\text {micro }}=d\left(E_{\text {intr }}\right)_{\text {micro }}
$$


The superscript $e$ at $Q$ means that heat is external, i.e., got from external heat sources according to the heat exchange laws (in microscopic way).

Heat (i) as system energy change in the appropriate process is usually calculated using heat capacity or entropy, while that (ii) as transferred energy in the heat exchange processes is usually calculated using heat fluxes determined from the corresponding equations of the heat exchange theory (Fourier, Newton-Richman et al.). Taking into account the proposed concepts of heat and work, the energy Eq. (41) becomes

$$
\mathrm{d} E_{\mathrm{intr}}=\mathrm{d} E_{\mathrm{k}}+\mathrm{d} U=\delta Q^{\mathrm{e}}+\sum \delta W^{\prime}{ }_{i}
$$

The work made by gravity forces (the motion energy transferred to the system particles because of their collisions with graviton fluxes) is equal to the loss of potential energy of mobile medium element position:

$$
\delta W_{\text {grav }}^{\prime}=\vec{F}_{\text {grav }} \mathrm{d} \vec{s}=-\mathrm{d} E_{\mathrm{p}}=-m g \mathrm{~d} z .
$$

By transposing this quantity to the left, we get in the left side of Eq. (45) a change of the total energy of a mobile medium element that is equal to the total change of intrinsic (internal and kinetic) and external (potential) energies:

$$
\begin{aligned}
\mathrm{d} E=\mathrm{d}\left(U+E_{\mathrm{k}}+E_{\mathrm{p}}\right)=\mathrm{d}\left(u+c^{2} / 2+g z\right) m= \\
=\delta Q^{\mathrm{e}}+\sum \delta W^{\prime}{ }_{i}=\delta Q^{\mathrm{e}}-\sum \delta W_{i} .
\end{aligned}
$$

Here $W=-W$ is intrinsic (with the minus sign) work because it is made by the internal system forces over the external bodies and is equal to the loss of intrinsic (internal) energy of the system (increase of the energy of external bodies).

Therefore, the authors who use as the basis the work $\delta W=\delta W^{\mathrm{v}}=p \mathrm{~d} V$ deal with the intrinsic (with the minus sign) work, while the authors who use as the basis the work $\delta W^{\prime}=\delta W^{\prime \mathrm{v}}=-p \mathrm{~d} V$ deal with the external (with the plus sign) work [21].

Equation (47) is identical to Eq. (2), so it is a general analytic expression of FLT for a flux. It also follows from the above method of derivation of this equation (based on the molecular-kinetic concept of matter structure) that the potential energy of external fields is not the intrinsic energy of system particles in their absolute motion relative to the stationary canal walls. 


\section{Conclusion}

In this work, the following investigation has been performed:

1. A critical analysis of obtaining the energy equations in thermodynamics was made.

2. It is proposed to use the symbols $\delta Q$ and $\delta W$ (rather than "imprecise, inexact differentials" of heat and work) to designate elementary character of the quantities heat and work and to call them "elementary heat" and "elementary work".

3. It is not recommended to use the symbols $d, D$ and $\delta$ as designations of differentials of heat and work because any differential literally means a difference of adjacent values of heat or work (that does not occurs in practice as a matter of fact).

4. A method is proposed for obtaining the FLT equation by applying the Newtonian mechanics to the own particles of the system under consideration. It is shown that no particles of different (gravitational, electric, etc.) fields are among the previous ones.

5. A strict interpretation of the physical quantities heat and work is given in the course of realization of the proposed method.

\section{References}

[1] C. Borgnakke, R.E. Sonnag, Fundamentals of Thermodynamics. University of Michigan: Willey, (2009), 894.

[2] B. Callen Thermodynamics and Introduction to Thermostatics, John Wiley and Sons, New York, (1985), 524.

[3] R. Hoyst, A. Poniewierski, Thermodynamics for Chemists, Physicists and Engineers. Science+Business Media, Dordrecht, (2012), 343.

[4] Ch. Kittel, Thermal Physics. John Willey and Sons, New York, (1973), 335.

[5] D. Kondepudi, I. Prigogine, Modern Thermodynamics: From Heat Engines to Dissipative Structures, John Wiley and Sons, New Delhi, India, (2015), 524.

[6] B.H. Lavenda, A New Perspective on Thermodynamics, Springer Science+Business Media, LLC, (2010), 219.

[7] M.J. Moran, H.N. Shapiro, D.D. Boettner, M.B. Balley, Fundamentals of Engineering Thermodynamics, John Wiley and Sons, USA, (2014), 553.

[8] F. Reyf, Statistical Physics: Berkeley Physics Course, the University of Berkeley, McGraw Hill Book Company, 5, (1972), 354.

[9] R. Morales-Rodriguez, Thermodynamics: Fundamentals and Its Application in Science, Technical University of Denmark, Denmark, (2016), 542. 
[10] R.C. Srivastava, K.S. Subit, K.J. Abhay, Thermodynamics: A Core Course, Prentice-Hall of India Private Limited, New Delhi, (2007), 274.

[11] H.D Baehr, Thermodynamics: An Introduction to the Basics and Their Technical Applications. 3. Reworked edition, Springer-Verlag, Berlin-Heidelberg-New York, (1973), 518.

[12] I.I. Novikov, Thermodynamics, Mashinostroenie, Moscow, (1984), 592.

[13] V.A. Kirillin, V.V. Sychev, A.E. Sheyndlin, Technical Thermodynamics, Energoatomizdat, Moscow, (1983), 448.

[14] V.I. Krutov, S.I. Isaev, A.I. Kozhinov et al., Technical Thermodynamics, Vysshaya Shkola, Moscow, (1991), 384.

[15] I.P. Bazarov, Thermodynamics, Vysshaya Shkola, Moscow, (1991), 376.

[16] L.C. Mazur, Technical Thermodynamics and Heat Engineering, GEOTAR-MED, Moscow, (2003), 352.

[17] N.M. Bazhin, V.A. Ivanchenko, V.N. Parmon, Thermodynamics for Chemists, Khimiya, Koloss, Moscow, (2004), 416.

[18] V.N. Lukanin, M.G. Shatrov, G.M. Kamfer et al., Heat Engineering, Vysshaya Shkola, Moscow, (2000), 671.

[19] M.P. Vukalovich, I.I. Novikov, Technical Thermodynamics, Energiya, Moscow, (1968), 495.

[20] V.I. Krutov, A.M. Arkharov, S.I. Isaev et al., Heat Engineering, Mashinostroenie, Moscow, (1986), 432.

[21] V.V. Ryndin, Philosophical and Physical Aspects of the First Law of Thermodynamics, LAP LAMBERT Academic Publishing, Saarbrucken, Germany (2015), 563.

[22] V.V. Ryndin, Heat Engineering, Kereku, Pavlodar, (2007), 460.

[23] L.G. Loitsianskii, Mechanics of Liquid and Gas, Drofa, Moscow, (2003), 840.

[24] J. Orear, Fundamental Physics, Cornell University, John Wiley, New York (1967), 556.

[25] L.D. Landau, E.M. Lifshitz, Fluid Mechanics (Vol. 6 of Course of Theoretical Physics), Nauka, Moscow, 6 (1988), 736. 
